\title{
Fetal metabolic programming and epigenetic modifications: a systems biology approach
}

\author{
Silvia Sookoiann ${ }^{1}$, Tomas Fernández Gianotti ${ }^{2}$, Adriana L. Burgueño ${ }^{2}$ and Carlos J. Pirola ${ }^{2}$
}

A growing body of evidence supports the notion that epigenetic changes such as DNA methylation and histone modifications, both involving chromatin remodeling, contribute to fetal metabolic programming. We use a combination of gene-protein enrichment analysis resources along with functional annotations and protein interaction networks for an integrative approach to understanding the mechanisms underlying fetal metabolic programming. Systems biology approaches suggested that fetal adaptation to an impaired nutritional environment presumes profound changes in gene expression that involve regulation of tissue-specific patterns of methylated cytosine residues, modulation of the histone acetylation-deacetylation switch, cell differentiation, and stem cell pluripotency. The hypothalamus and the liver seem to be differently involved. In addition, new putative explanations have emerged about the question of whether in utero overnutrition modulates fetal metabolic programming in the same fashion as that of a maternal environment of undernutrition, suggesting that the mechanisms behind these two fetal nutritional imbalances are different. In conclusion, intrauterine growth restriction is most likely to be associated with the induction of persistent changes in tissue structure and functionality. Conversely, a maternal obesogenic environment is most probably associated with metabolic reprogramming of glucose and lipid metabolism, as well as future risk of metabolic syndrome (MS), fatty liver, and insulin (INS) resistance.

$\mathbf{L}^{2}$ arge amounts of epidemiological data showed that impaired intrauterine growth and adult metabolic and cardiovascular disorders, including coronary heart disease, type 2 diabetes, and insulin (INS) resistance, are strongly associated (1-4). Actually, the first exploration of a putative connection between environmental influence in early life and the risk of cardiovascular disease in adulthood was done by David Barker and coworkers, who followed-up a cohort of 499 men and women born in Preston (Lancashire, UK) during 1935-1943, and observed that as adults the highest blood pressures occurred in people who had been small babies with large placentas (5).

Of note, the concept of a relationship between birth weight and adult chronic diseases initially described in low-birthweight babies was further extended to large-for-gestational age (LGA) babies, and the term of inappropriate gestational weight gained acceptance to better illustrate changes in earlylife metabolic environment that contribute to the risk of metabolic syndrome (MS) in adulthood (6).

Barker and Hales also provided the initial answer to the question of how birth weight and adult chronic diseases are connected. They explained that fetuses adapt to an impaired supply of nutrients by changing their physiology and metabolism, and altering the sensitivity of tissues causes an abnormal structure and function in adult life; thereby, they formulated the "thrifty phenotype hypothesis" $(7,8)$.

Altogether, the above-mentioned clinico-epidemiological evidence strongly suggests a close interaction between fetal and maternal environment and modulation of gene expression that starts very early in life and can even be passed across generations. Hence, the notion about epigenetic modifications such as DNA methylation and covalent posttranslational histone modifications, which mediate phenomena such as genomic imprinting and chromatin remodeling, emerged as the most suitable molecular explanation of fetal metabolic programming.

In this review, we integrate genomic, molecular, and physiological data to explore the putative interplay between the underlying genetic and epigenetic mechanisms involved in fetal metabolic programming to better understand how they might be associated with adult disease. This approach is based on the hypothesis that a more integrative knowledge of the genetic/epigenetic determinants of fetal origin of adult diseases may have a strong impact on interventional programs and putative emerging therapies.

We also contrast the hypotheses about impaired nutrient availability during the fetal period being associated with different biological processes depending on fetus exposure to a maternal protein/nutrient restriction or an overnutrition/ high-fat (HF) maternal environment.

Moreover, we discuss whether fetal metabolic programming is controlled by metabolically active target tissues such as the liver, or contrarily, is modulated by central neural pathways involved in appetite and energy balance regulation, such as those involving the hypothalamus. In addition, we introduce the concept of "fetal mitochondrial programming" of adult chronic diseases.

Finally, we show new data about the role of chromatin remodeling and illustrate areas of future research that remain

'Department of Clinical and Molecular Hepatology, Institute of Medical Research A Lanari-IDIM, University of Buenos Aires, National Council of Scientific and Technological Research (CONICET), Ciudad Autónoma de Buenos Aires, Argentina; ${ }^{2}$ Department of Molecular Genetics and Biology of Complex Diseases, Institute of Medical Research A Lanari-IDIM, University of Buenos Aires, National Council of Scientific and Technological Research (CONICET), Ciudad Autónoma de Buenos Aires, Argentina. Correspondence: Carlos J. Pirola (pirola.carlos@lanari.fmed.uba.ar) 
\begin{tabular}{l|l} 
Review & Sookoian et al.
\end{tabular}

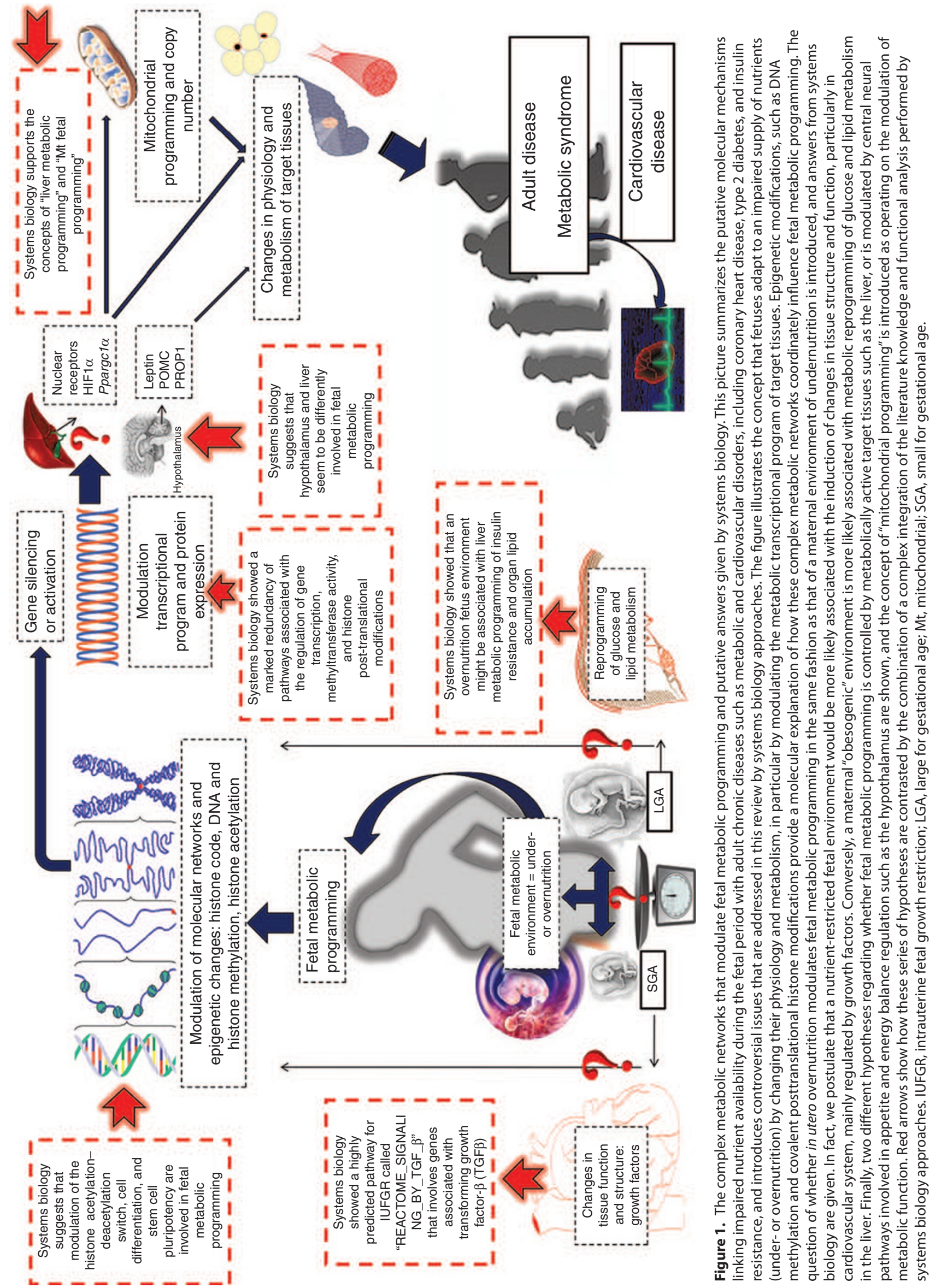


poorly explored, such as the role of microRNAs (miRNAs) in the regulation of fetal metabolic programming.

A summary diagram that guides the readers as to how these complex metabolic networks may be perturbed by genomic and epigenetic changes and how these hypotheses are linked to our findings is shown in Figure 1.

\section{SYSTEMS BIOLOGY APPROACH AND GENE-REGULATORY NETWORK ANALYSIS TO UNDERSTAND FETAL METABOLIC PROGRAMMING IN AN INTEGRATIVE FASHION}

Systems biology approaches based on the combinations of omics (genomics, proteomics, or metabolomics) data, for example, expression array analysis, the measurement of transcript levels, gene-gene and protein-protein interactions, and the relation between transcript levels and clinical traits, might be able to decipher the disease pathways or molecular mechanisms underlying the fetal metabolic programming that is associated with adult chronic diseases. Hence, we chose systems biology to explain fetal metabolic programming because it introduces a new and integrative concept of the pathogenesis of human disorders and suggests the presence of common physiologic processes and molecular networks influencing the risk of a disease.

Our approach to contrast this hypothesis involved the use of a text-mining tool for collecting the available evidence about fetal metabolic programming in a systematic manner that further allows us to predict biomolecular interactions among the genes/proteins. Therefore, we used the Platform for Exploration of Significant Concepts Associated to Co-occurrences
Relationships (PESCADOR) (9) with the query "(fetal programming OR newborn body weight OR small for gestational age OR SGA OR large for gestational age) and (epigenetics OR DNA methylation or histone)." PESCADOR allows the selection of gene/protein co-occurrence pairs based on their relatedness to biological concepts, bringing together, under a common perspective, protein interactions that have not been studied under the same research focus (9). After abstract tagging, 699 gene/ protein terms were identified in 942 published abstracts. Of note, when these terms and interactions were displayed graphically, a hierarchical central hub appears centered on two gene/ proteins: histone deacetylases and DNA cytosine-5-methyltransferases (Figure 2). Even more attractive is the prediction of interconnected hubs under the influence of histone deacetylases and histone demethylases that specifically demethylate "Lys-4" of histone $\mathrm{H} 3$ (H3K4me1-3), such as the histone demethylase Jumonji/AT-rich interactive domain-containing protein 1A, which modulates the histone code during cell differentiation, and octamer-binding protein 4 (OCT4, also known as POU5F1), which along with NANOG, is involved in early embryogenesis and stem cell pluripotency through modulation of DNA methylation (10). At the same level of significance are also placed BRG1 (alias SWI/SNF-related, matrix-associated, and actindependent regulator of chromatin), which is a transcriptional coactivator that cooperates with nuclear hormone receptors to potentiate transcriptional activation; CTCF (CCCTC-binding factor-zinc finger protein), involved in transcriptional regulation by binding to chromatin insulators and preventing interaction between promoter and nearby enhancers and silencer; and

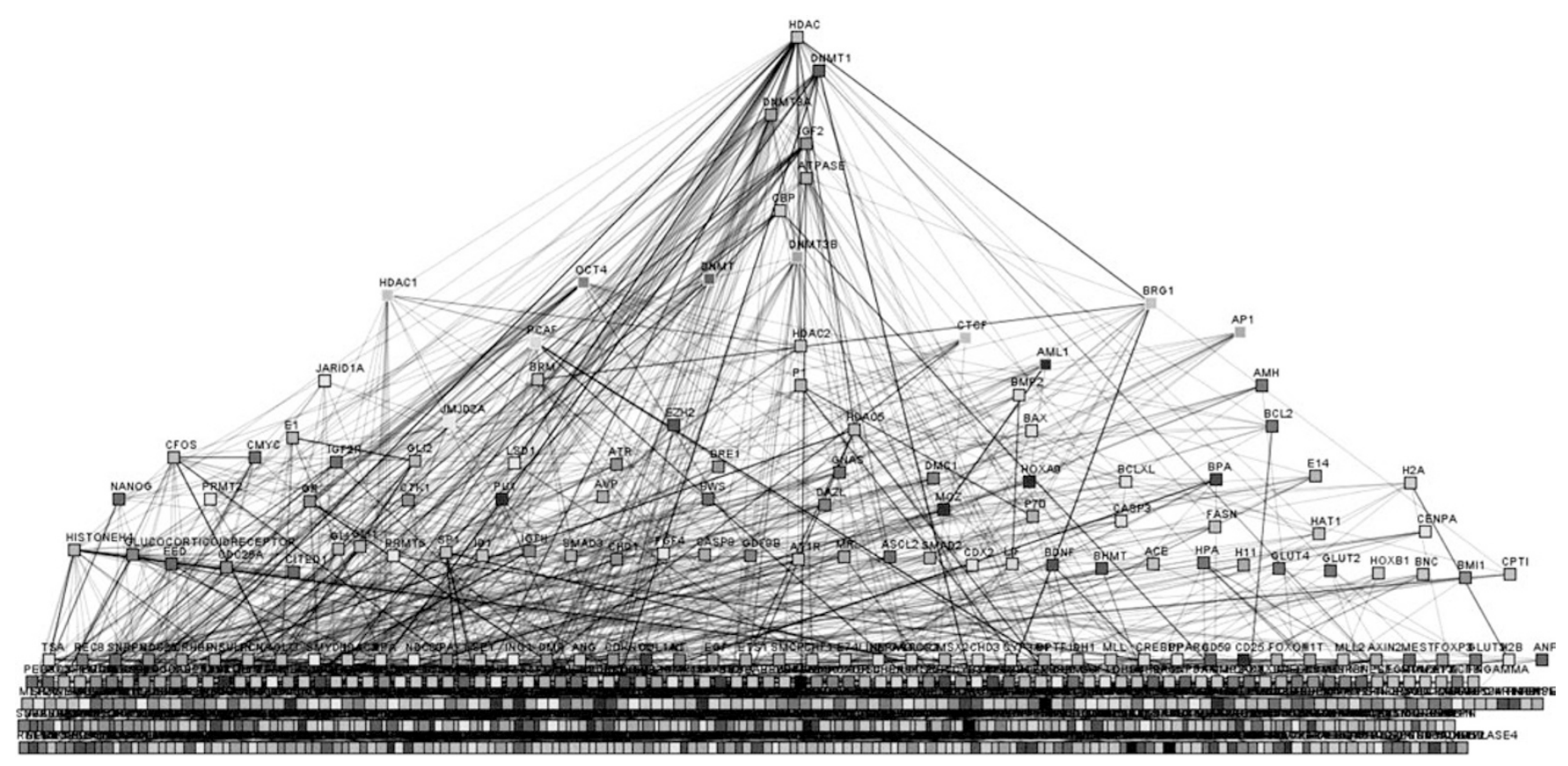

Figure 2. Gene/protein co-occurrence and their relatedness to biological concepts with the query "(fetal programming OR newborn body weight OR small for gestational age OR SGA OR large for gestational age OR LGA) and (epigenetics or DNA methylation or histone)". Prediction was performed by PESCADOR (available at http://cbdm.mdc-berlin.de/tools/pescador/), a web-based tool to assist large-scale integration text mining of biointeractions extracted from MEDLINE abstracts. The graph was constructed using the freely available program MEDUSA, which is a Java application for visualizing and manipulating graphs of interaction (http://www.bork.embl.de/medusa) (refs. 10 and 49). PESCADOR, Platform for Exploration of Significant Concepts Associated to Co-occurrences Relationships. 


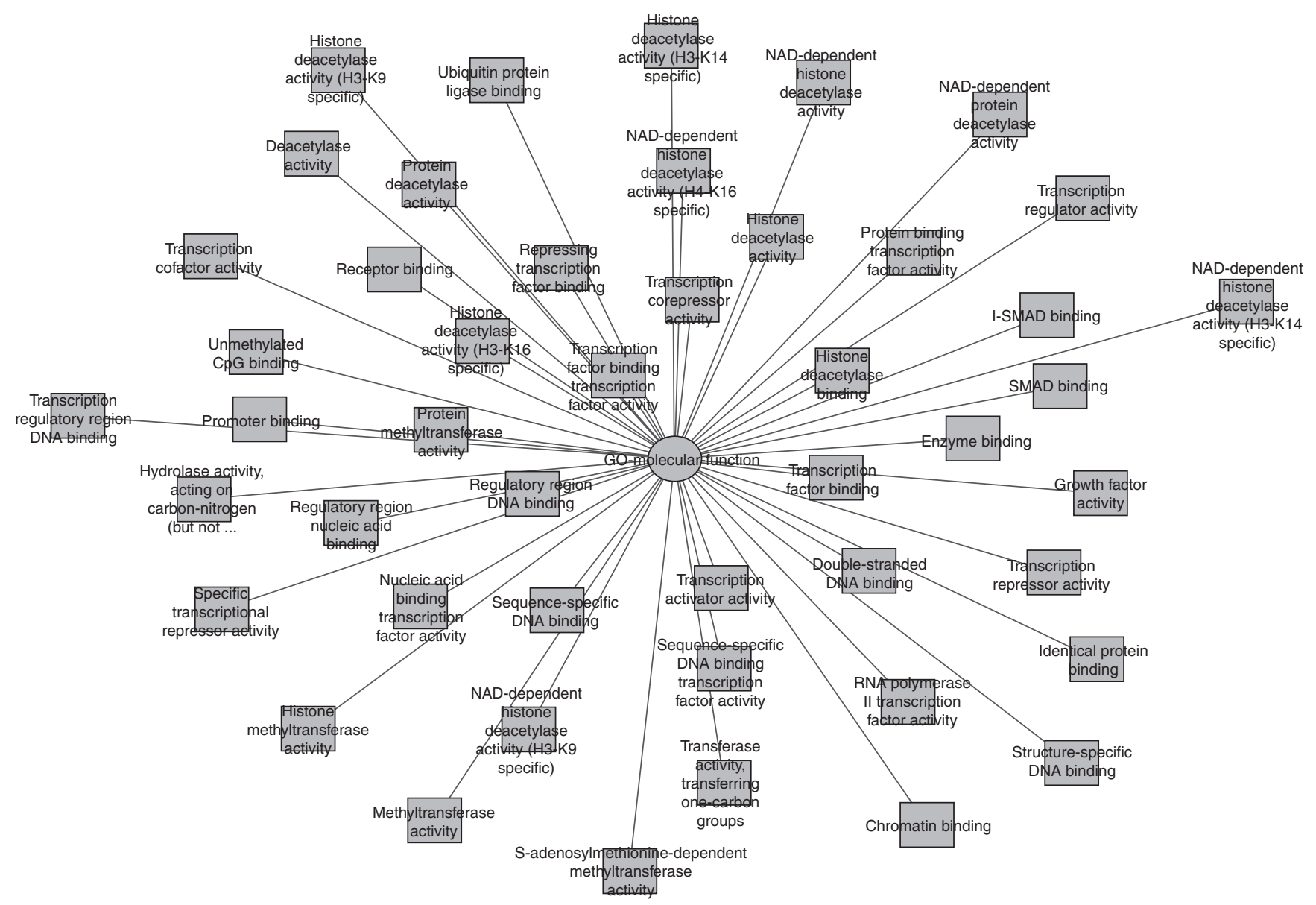

Figure 3. Results of functional association analysis performed by the bioinformatics resource GenMANIA (available at http://www.genemania.org/, Toronto, Canada). Illustration includes molecular functions summarized in gene ontology (GO) terms (the GO pathway) based on a set of input genes identified by the text-mining tool PESCADOR (Supplementary Appendix S1 online). The network is shown as a cytoscape graph generated from ToppCluster (available at http://toppcluster.cchmc.org/) network analysis. PESCADOR, Platform for Exploration of Significant Concepts Associated to Co-occurrences Relationships.

DNA cytosine-5-methyltransferase $3 \beta$, required for genomewide de novo methylation and involved in gene silencing. In addition, highly ranked are several glucose transporters (GLUT2 and GLUT4), several renin-angiotensin-aldosterone system components (ACE and AT1R), and unsurprisingly, growth factors or their receptors, and so forth.

Furthermore, based on the candidate gene list identified by the text-mining tool PESCADOR (Supplementary Appendix S1 online), we performed a functional association analysis by the bioinformatics resource GenMANIA (11), which includes protein and genetic interactions, pathways, coexpression, colocalization, and protein domain similarity. Figure 3 shows the results of molecular functions predicted from the gene list, summarized in gene ontology (GO) terms (the GO pathway). As expected, there is a marked redundancy of pathways associated with the regulation of gene transcription, methyltransferase activity, histone posttranslational modifications, such as histone acetylation and chromatin remodeling.

In conclusion, fetal adaptation to an abnormal nutritional environment presumes profound changes in gene expression that involves not only regulation of tissue-specific patterns of methylated cytosine residues by DNA cytosine-5methyltransferases, but also modulation of the histone acetylation and deacetylation switch, cell differentiation, and stem cell pluripotency with probable implications on further development of organs and gene expression of key components of inflammatory, metabolic, and cardiocirculatory pathways.

MATERNAL ENVIRONMENT AND FETAL PROGRAMMING: UNDERNUTRITION VS. OVERNUTRITION: DO THEY SHARE THE SAME BIOLOGICAL PATHWAYS?

Initial epidemiological studies showed that fetal undernutrition during gestation was associated with increased mortality owing to ischemic heart disease in adulthood and higher risk of suffering from the cluster of diseases that integrate the MS (4). Of note, a meta-analysis that included 14 studies and a total of 132,180 individuals showed that low $(<2,500 \mathrm{~g})$ and also high birth weight $(>4,000 \mathrm{~g})$ are associated with increased risk of type 2 diabetes, indicating that birth weight and laterlife risk of type 2 diabetes can be illustrated in a $\mathrm{U}$-shaped relation (2). Nevertheless, whether fetal programming by maternal obesity involves the same mechanisms as that of maternal undernutrition is still unknown. 
Table 1. Functional enrichment analysis of candidate genes with previous evidence about association between fetal metabolic programming and intrauterine growth restriction

\begin{tabular}{|c|c|c|c|c|}
\hline Identification number & Name/source & $P$ value & $\begin{array}{l}\text { Term in } \\
\text { query }\end{array}$ & $\begin{array}{l}\text { Term in } \\
\text { genome }\end{array}$ \\
\hline \multicolumn{5}{|l|}{ GO: molecular function } \\
\hline GO:0043565 & Sequence-specific DNA binding & $1.317 \times 10^{-7}$ & 11 & 693 \\
\hline GO:0051718 & DNA (cytosine-5-)-methyltransferase activity, acting on CpG substrates & $3.862 \times 10^{-7}$ & 3 & 3 \\
\hline GO:0008134 & Transcription factor binding & $1.442 \times 10^{-6}$ & 8 & 323 \\
\hline GO:0003886 & DNA (cytosine-5-)-methyltransferase activity & $1.543 \times 10^{-6}$ & 3 & 4 \\
\hline \multicolumn{5}{|l|}{ GO: biological process } \\
\hline GO:0016481 & Negative regulation of transcription & $2.340 \times 10^{-9}$ & 13 & 666 \\
\hline GO:0045892 & Negative regulation of transcription, DNA dependent & $3.327 \times 10^{-9}$ & 12 & 523 \\
\hline GO:0051253 & Negative regulation of RNA metabolic process & $3.888 \times 10^{-9}$ & 12 & 530 \\
\hline GO:0009892 & Negative regulation of metabolic process & $4.703 \times 10^{-9}$ & 15 & 1,112 \\
\hline GO:0005694 & Chromosome & $5.594 \times 10^{-5}$ & 8 & 555 \\
\hline GO:0000792 & Heterochromatin & $1.314 \times 10^{-4}$ & 4 & 61 \\
\hline GO:0000775 & Chromosome, centromeric region & $1.822 \times 10^{-4}$ & 5 & 153 \\
\hline GO:0005667 & Transcription factor complex & $4.210 \times 10^{-4}$ & 6 & 324 \\
\hline \multicolumn{5}{|l|}{ Mouse phenotype (MP) } \\
\hline MP:0002981 & Increased liver weight & $3.341 \times 10^{-4}$ & 6 & 77 \\
\hline MP:0004848 & Abnormal liver size & $7.613 \times 10^{-4}$ & 9 & 299 \\
\hline MP:0000599 & Enlarged liver & $1.724 \times 10^{-3}$ & 7 & 163 \\
\hline MP:0008907 & Decreased total fat pad weight & $6.683 \times 10^{-3}$ & 3 & 10 \\
\hline PW:0000490 & TGF- $\beta$ Smad-dependent signaling/pathway ontology & $2.545 \times 10^{-4}$ & 4 & 26 \\
\hline
\end{tabular}

The analysis was done based on transcriptome, proteome, regulome (transcription factor binding sites and miRNA), ontologies (the gene ontology (GO) pathway), phenotype (human disease and mouse phenotype), pharmacome (drug-gene associations), literature cocitation, and other features by the bioinformatics resource ToppGene Suite (http://toppgene.cchmc.org). KEGG, Kyoto Encyclopedia of Genes and Genomes; miRNA, microRNA; TGF- $\beta$, transforming growth factor- $\beta$.

Hence, we wondered whether the biological processes and the disease pathways under these two opposing fetal environments are similar or, to the contrary, if they significantly differ. To answer this question, we used a text-mining tool for generating regulatory pathways associated with both possible scenarios: an environment of undernutrition (from either maternal protein restriction or placental insufficiency) and the opposite, obesogenic environment.

Text mining was done by PESCADOR under the same abovementioned query but restricting the search by adding either the terms "(intrauterine fetal growth restriction OR IUFGR OR (maternal and (caloric restriction OR protein restriction)))" for fetal undernutrition query or the terms "(maternal and (obesity OR high-fat diet OR HFD)" for the obesogenic environment. The tagged abstracts showed a list of 28 genes/proteins associated with IUFGR and 9 genes/proteins associated with HF maternal diet during pregnancy, Supplementary Appendix S1 online.

Functional enrichment analysis, shown in Tables 1 and 2, was performed by the bioinformatics resource ToppGene Suite (http://toppgene.cchmc.org, Cincinnati, $\mathrm{OH}$ ) based on transcriptome, proteome, regulome (transcription factor binding sites and miRNA), ontologies (GO, pathway), phenotype (human disease and mouse phenotype), pharmacome (druggene associations), literature cocitation, and other features. Of note, the analysis showed that the reported loci for IUFGR were integrated into several common functional pathways and biological processes (Table 1) that significantly differ from those of the reported loci for maternal HFD (Table 2). For instance, the predicted biological processes for IUFGR are mainly 
Table 2. Functional enrichment analysis of candidate genes with previous evidence about association between fetal metabolic programming and maternal high-fat diet or obesity

\begin{tabular}{|c|c|c|c|c|}
\hline $\begin{array}{l}\text { Identification } \\
\text { number }\end{array}$ & Name/source & $P$ value & $\begin{array}{l}\text { Term in } \\
\text { query }\end{array}$ & $\begin{array}{l}\text { Term in } \\
\text { genome }\end{array}$ \\
\hline \multicolumn{5}{|c|}{ GO: molecular function } \\
\hline GO:0005179 & Hormone activity & $1.707 \times 10^{-3}$ & 3 & 112 \\
\hline GO:0005184 & $\begin{array}{l}\text { Neuropeptide } \\
\text { hormone activity }\end{array}$ & $7.123 \times 10^{-3}$ & 2 & 27 \\
\hline GO:0005102 & Receptor binding & $7.171 \times 10^{-3}$ & 5 & 1,097 \\
\hline GO:0008134 & $\begin{array}{l}\text { Transcription factor } \\
\text { binding }\end{array}$ & $3.959 \times 10^{-2}$ & 3 & 323 \\
\hline GO:0031841 & $\begin{array}{l}\text { Neuropeptide } \\
\text { Y-receptor binding }\end{array}$ & $4.745 \times 10^{-2}$ & 1 & 1 \\
\hline \multicolumn{5}{|c|}{ GO: biological process } \\
\hline GO:0042593 & $\begin{array}{l}\text { Glucose } \\
\text { homeostasis }\end{array}$ & $2.155 \times 10^{-5}$ & 4 & 73 \\
\hline GO:0033500 & $\begin{array}{l}\text { Carbohydrate } \\
\text { homeostasis }\end{array}$ & $2.155 \times 10^{-5}$ & 4 & 73 \\
\hline GO:0006094 & Gluconeogenesis & $1.700 \times 10^{-3}$ & 3 & 56 \\
\hline GO:0045834 & $\begin{array}{l}\text { Positive regulation } \\
\text { of lipid metabolic } \\
\text { process }\end{array}$ & $2.799 \times 10^{-3}$ & 3 & 66 \\
\hline GO:0019319 & $\begin{array}{l}\text { Hexose } \\
\text { biosynthetic } \\
\text { process }\end{array}$ & $2.930 \times 10^{-3}$ & 3 & 67 \\
\hline \multicolumn{5}{|c|}{ GO: cellular component } \\
\hline GO:0017053 & $\begin{array}{l}\text { Transcriptional } \\
\text { repressor complex }\end{array}$ & $1.189 \times 10^{-2}$ & 2 & 53 \\
\hline GO:0044451 & Nucleoplasm part & $1.333 \times 10^{-2}$ & 4 & 783 \\
\hline \multicolumn{5}{|c|}{ Mouse phenotype (MP) } \\
\hline MP:0011174 & Lipodystrophy & $2.622 \times 10^{-3}$ & 2 & 3 \\
\hline MP:0001363 & $\begin{array}{l}\text { Increased anxiety- } \\
\text { related response }\end{array}$ & $5.363 \times 10^{-3}$ & 4 & 123 \\
\hline MP:0002628 & Hepatic steatosis & $8.727 \times 10^{-3}$ & 4 & 139 \\
\hline MP:0009135 & $\begin{array}{l}\text { Abnormal brown } \\
\text { fat cell size }\end{array}$ & $1.308 \times 10^{-2}$ & 2 & 6 \\
\hline MP:0001362 & $\begin{array}{l}\text { Abnormal anxiety- } \\
\text { related response }\end{array}$ & $2.943 \times 10^{-2}$ & 4 & 189 \\
\hline \multicolumn{5}{|l|}{ Pathway } \\
\hline hsa04920 & $\begin{array}{l}\text { Adipocytokine } \\
\text { signaling pathway; } \\
\text { KEGG pathway }\end{array}$ & $6.281 \times 10^{-3}$ & 3 & 67 \\
\hline
\end{tabular}

The analysis was done based on transcriptome, proteome, regulome (transcription factor binding sites and miRNA), ontologies (gene ontology (GO) pathway), phenotype (human disease and mouse phenotype), pharmacome (drug-gene associations), literature cocitation, and other features by the bioinformatics resource ToppGene Suite (http://toppgene.cchmc.org).

KEGG, Kyoto Encyclopedia of Genes and Genomes; miRNA, microRNA.

enriched by mechanisms of gene transcription and chromatin structure regulation and DNA (cytosine-5)-methyltransferase activity. Conversely, the predicted GO terms for maternal HFD during pregnancy are mainly enriched by mechanisms of cellular control of glucose and lipid and lipoprotein metabolism and hormone activity. An interesting finding that emerged from the analysis and that deserves follow-up is that of a highly predicted pathway for IUFGR called "REACTOME_ SIGNALING_BY_TGF_BETA" that involves genes associated with transforming growth factor- $\beta$ (Table 1 ). This finding might suggest that a nutrient-restricted fetal environment directly influences the development of the cardiovascular system, as supported by the epidemiological evidence (4). Thus, we might speculate that IUFGR is mostly predisposed to heartrelated diseases in adult life, including coronary heart disease.

By contrast, an overnutrition fetal environment might be associated with liver metabolic programming of INS resistance, and organ lipid accumulation as the "adipocytokine signaling pathway" was highly predicted for the reported loci associated with HF maternal diet.

On the other hand, the cellular compartments predicted from the candidate gene list for IUFGR are chromosome and heterochromatin, whereas for HFD during pregnancy, they were the transcriptional repressor complex and nucleoplasm (Tables 1 and 2).

Finally, an interesting finding emerged from the functional enrichment analysis predicted from the mouse models, i.e., an altered "liver phenotype." Curiously, this liver phenotype is associated with either abnormal liver weight and size or hepatic steatosis, and it was highly predicted for both IUFGR and an obesogenic environment. Of note, hepatomegaly and altered liver metabolism are hallmarks of a well-known model of cardiovascular diseasethe spontaneously hypertensive rat (12). This finding strongly suggests a critical role of "liver fetal metabolic programming" in adult MS-related phenotypes, which may explain the strong association between nonalcoholic fatty liver disease severity and atherosclerosis that we have recently shown (13).

In conclusion, it seems that IUFGR is more likely associated with the induction of persistent changes in tissue structure and function mainly regulated by growth factors such as transforming growth factor- $\beta 1$ or INS-like growth factors and their receptors. Conversely, a maternal obesogenic environment is more likely associated with metabolic reprogramming of glucose and lipid metabolism and future risk of MS and INS resistance.

\section{FETAL PROGRAMMING OF METABOLICALLY ACTIVETARGET TISSUES: COMMON MOLECULAR PATHWAYS OR SPECIFIC TISSUE-METABOLIC IMPRINTING?}

As mentioned before, the thrifty phenotype hypothesis proposed that fetal adaptations to poor nutrition in utero led to permanent changes in INS and glucose metabolism (7). Therefore, it is reasonable to speculate that metabolically active tissues such as the liver are key players in fetal metabolic programming (14-16). Conversely, it was also postulated that fetal metabolic programming is centrally regulated by hypothalamic proopiomelanocortin and neuropeptide $\mathrm{Y}$ genes, which are in turn regulated by leptin, which controls energy balance and appetite (17-19). Fetal leptin, however, is not a good candidate because as expected it correlates almost linearly with the newborn's body weight (20) and does not explain the risk associated with the two extremes of fetal growth. We present other alternatives below.

We wondered how strong the evidence is for each theory; hence, we used systems biology approaches to answer this question. By using text mining with the query "(fetal programming OR newborn body weight OR small for gestational age OR SGA OR large for gestational age OR LGA) and 

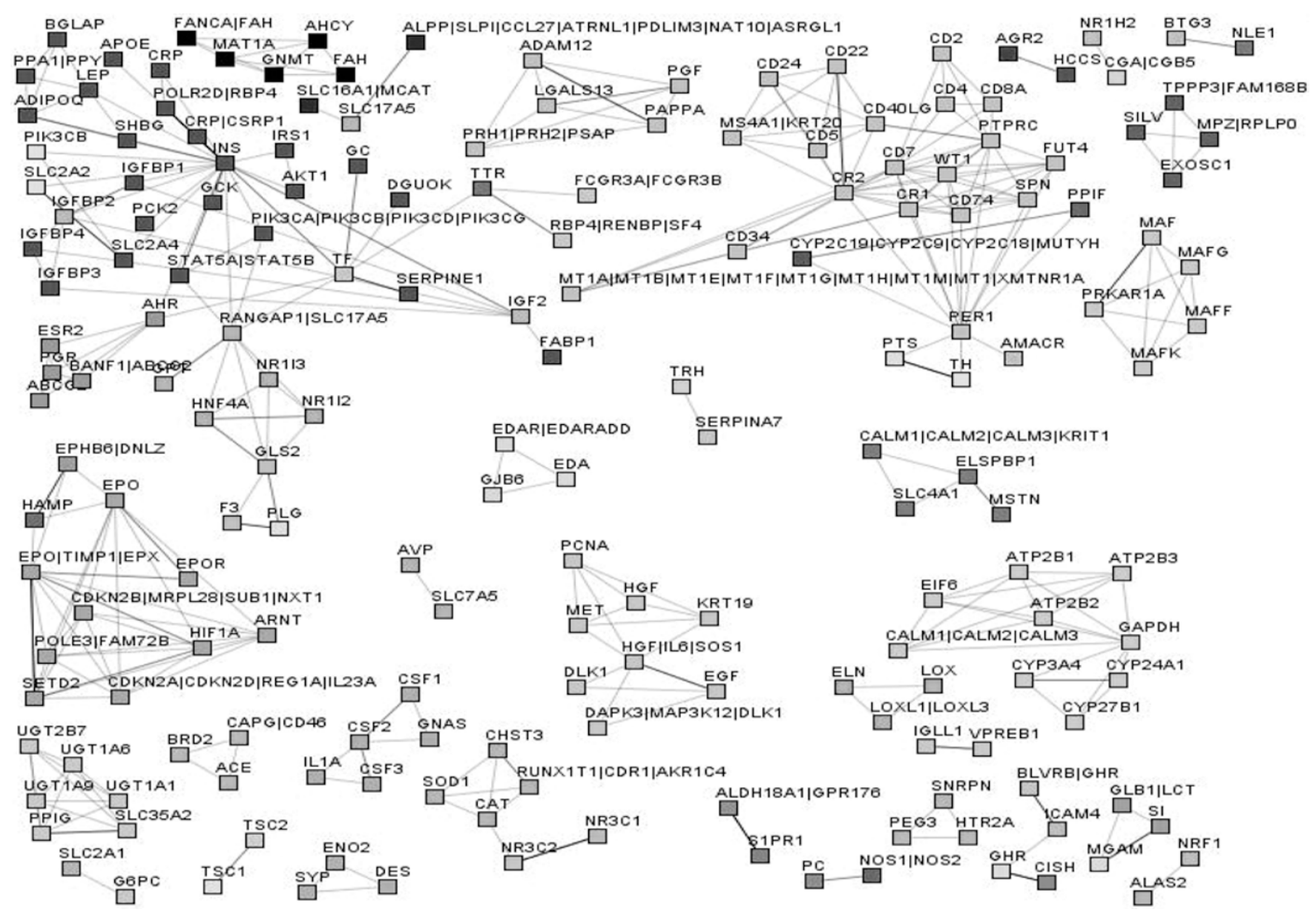

Figure 4. Graphic illustration of gene/protein co-occurrences and their relatedness to biological concepts with the query"(fetal programming OR newborn body weight OR small for gestational age OR SGA OR large for gestational age OR LGA) and (LIVER)." Prediction was performed by PESCADOR (available at http://cbdm.mdc-berlin.de/tools/pescador/), a web-based tool to assist large-scale integration text mining of biointeractions extracted from MEDLINE abstracts. Graph was constructed as in Figure 1. PESCADOR, Platform for Exploration of Significant Concepts Associated to Co-occurrences Relationships.

(liver)," 667 abstracts/papers were explored, and the number of co-occurrences retrieved was 530. The gene list is shown in Supplementary Appendix $\mathbf{S 2}$ online.

Of note, "liver metabolic programming" involves large networks of genes and proteins including but not restricted to INSrelated pathways (Figure 4). For instance, hypoxia-inducible factor-1 (HIF1 $\alpha$ and HIF1 $\beta$, alias ARNT), nuclear receptors (NR1H2, alias liver $\mathrm{X}$ receptor), and signal transducer and activator of transcription proteins, which are critical determinants of genetic susceptibility to fatty liver and mitochondrial liver function (12,21-23), and SERPINE1 (PAI1), involved in cardiovascular risk and MS (24), integrate highly predicted nodes.

Supporting this, the observation of our group showed that maternal HFD feeding during pregnancy is associated with a programming effect on the liver abundance of Ppargcla mRNA that predisposes the offspring to develop INS resistance and MS-related phenotypes when they are exposed to a metabolic insult in later life (14). At the same time, we demonstrated that the "liver metabolic imprinting" also programs liver mitochondrial DNA copy number (14). Moreover, we reported that both extremes of neonatal birth weight are associated with decreased umbilical cord mitochondrial DNA content (20).
It is worth noting that the concept of "mitochondrial programming," despite being unexplored, is remarkable as there is evidence from human studies that epigenetic mechanisms operate on mitochondrial function and critically modulate INS resistance and MS-related phenotypes by altering not only the nuclear but also the mitochondrial genome $(25,26)$. Indeed, the new concept of "mitochondrial epigenetics" is emerging.

Finally, in addition to INS itself, are several interesting candidate genes associated with MS components such as type 2 diabetes, including HNF4A (27). Others, such as PER1, constitute key components of the circadian mechanism, which controls the entire cell metabolism. Mutations in PER1 and other family members make mice more prone to gain weight under HFD (28), and normal variants of the $C L O C K$ promoter are associated with nonalcoholic fatty liver disease or obesity in humans $(29,30)$.

On the other hand, text mining with the query "(fetal programming OR newborn body weight OR small for gestational age OR SGA or large for gestational age OR LGA) and (hypothalamus)" showed 132 abstracts/papers and retrieved 228 cooccurrences; the gene list is shown in Supplementary Appendix S3 online. The analysis of the interaction between terms showed four central hubs centered on leptin, proopiomelanocortin, 


\section{Review | Sookoian etal.}
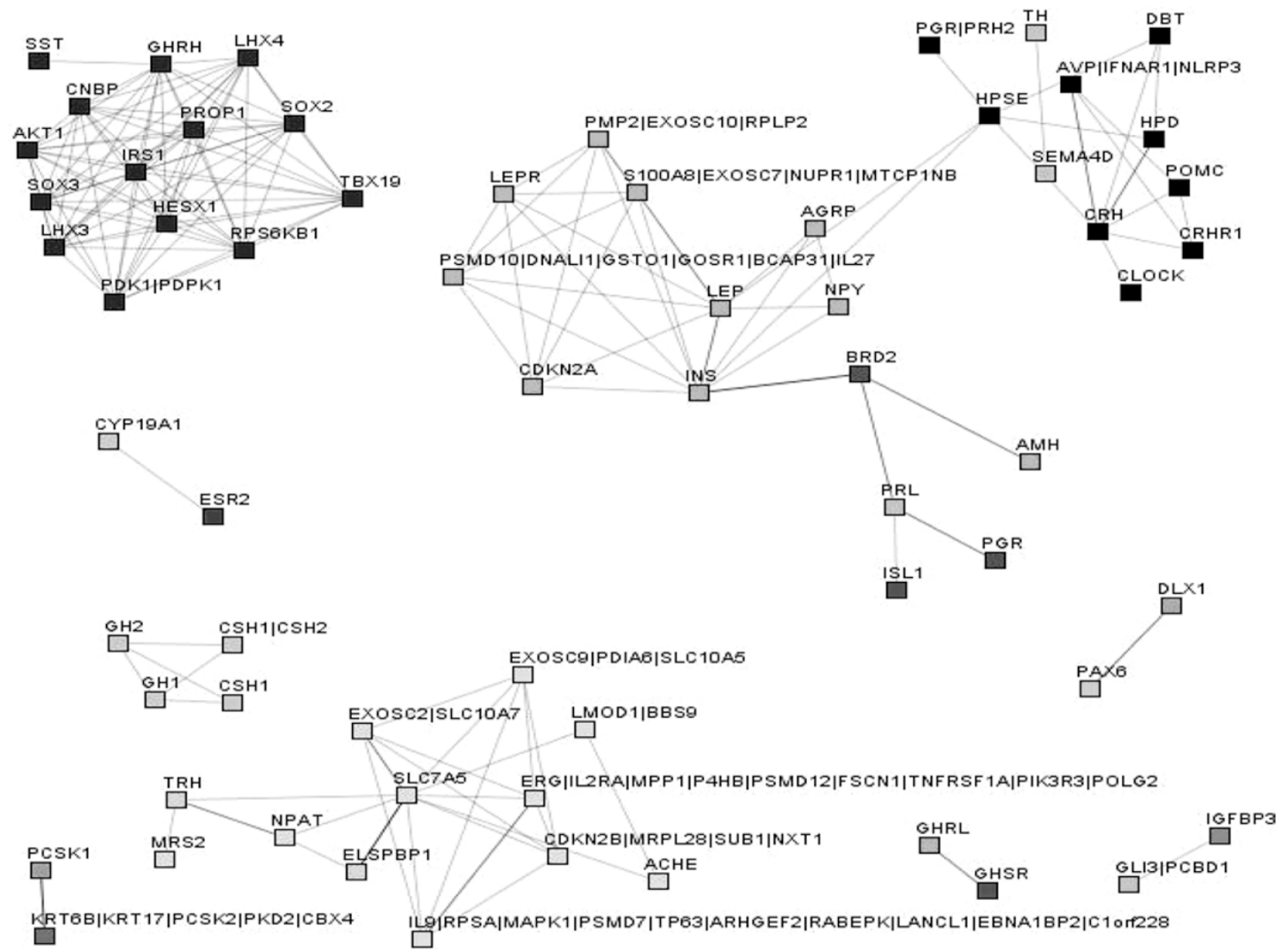

Figure 5. Graphic illustration of gene/protein co-occurrences and their relatedness to biological concepts with the query" (fetal programming OR newborn body weight OR small for gestational age OR SGA OR large for gestational age OR LGA) and (HYPOTHALAMUS)." Prediction was performed by PESCADOR (available at http://cbdm.mdc-berlin.de/tools/pescador/), a web-based tool to assist large-scale integration text mining of biointeractions extracted from MEDLINE abstracts. Graph was constructed as in Figure 1. PESCADOR, Platform for Exploration of Significant Concepts Associated to Co-occurrences Relationships.

INS receptor substrate 1, and PROP1 (possibly involved in the ontogenesis of pituitary gonadotropes) and SLC7A5, the solute carrier family 7 (amino acid transporter light chain L system) involved in cellular amino acid uptake (Figure 5). Two remarkable findings that were also found in the liver programming search are worth mentioning. One of them, the CLOCK gene, a master regulator of circadian function associated with MS in humans as already mentioned $(29,30)$ and rodent studies (31), was connected to corticotropin releasing hormone and proopiomelanocortin-related nodes. The second one, the SLC7A5-related nodes, predicted thyrotropin-releasing hormone, which was associated with hypertension, obesity-related hypertension, and control of body weight in rodents $(32,33)$ and in humans (34).

In line with these observations, we explored in a rat model, the impact of developmental and long-term HFD on DNA methylation of a number of diencephalic candidate genes and one gene, a member of the zinc finger family of proteins, called zinc finger protein 91 homolog (ZFP91) was particularly altered. The protein encoded by ZFP91 is a potent survival factor for neurons and also acts as a regulator of the noncanonical NF- $\mathrm{\beta} \beta$ pathway. In addition, a read-through transcript variant composed of ZFP91 and the downstream ciliary neurotrophic factor $(C N T F)$ gene, an endogenous modulator of energy homeostasis in the arcuate nucleus (35), has been reported. We observed that male offspring of HFD-fed dams, which were born large according to the mean weight of their littermates (above 80th percentile), had higher levels of DNA methylation in the ZFP91 promoter (data not shown). Hence, we might speculate that methylation of ZFP91-CNTF during fetal life is associated with impairment of central regulation of energy homeostasis and food intake, leading to diet-induced obesity in adult life. Of note, ciliary neurotrophic factor may regulate mitochondrial function by upregulating transcription factor A, mitochondrial (TFAM) (36). As mentioned later, altered increased DNA methylation of the TFAM promoter and decreased mitochondrial DNA copy number were found in adolescents with MS components, in particular INS resistance $(37,38)$.

In conclusion, exposure to a nutritional insult in early life modulates the functionality of metabolically active target tissues such as the liver, involving a complex network of gene 


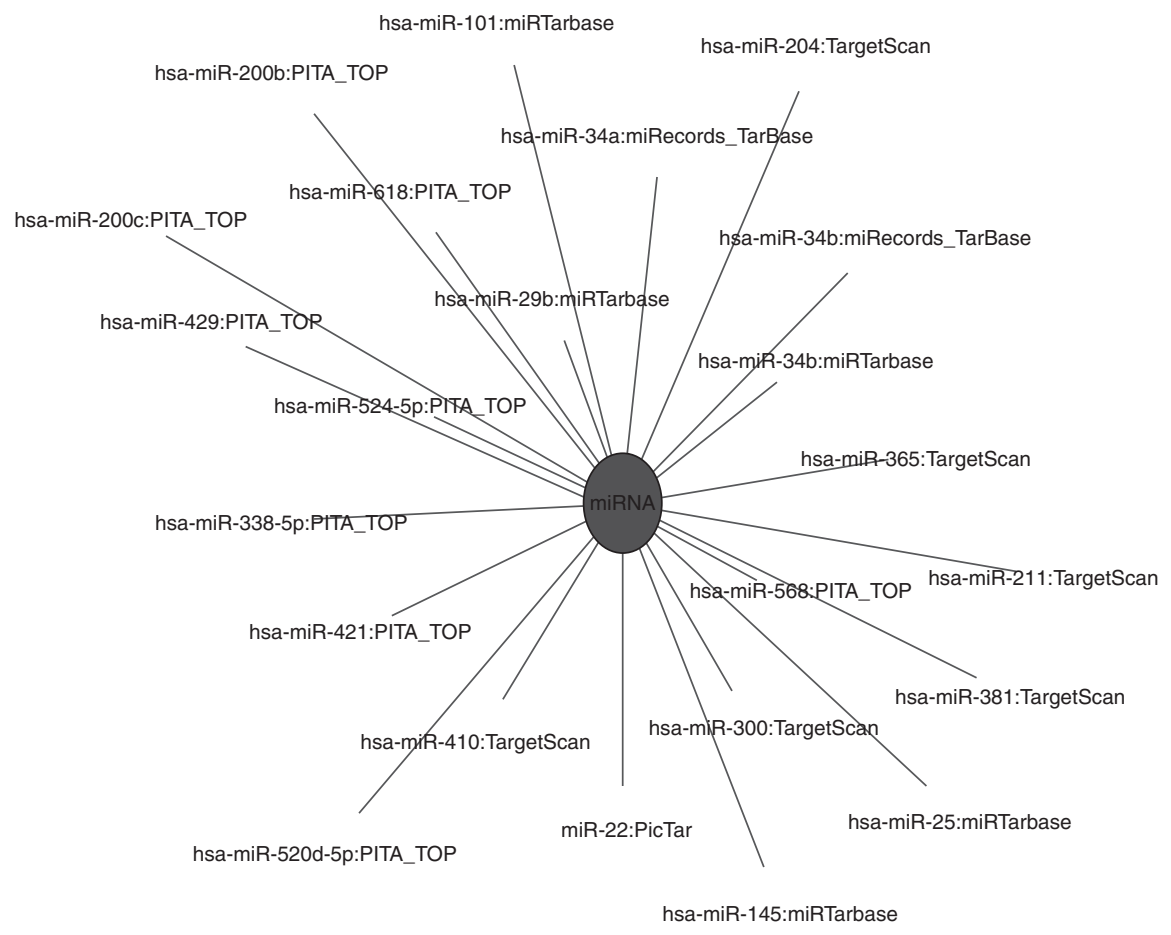

Figure 6. Functional enrichment analysis of putative miRNAs associated with fetal metabolic programming. The network is shown as a cytoscape graph generated from ToppCluster (available at http://toppcluster.cchmc.org/) network analysis. miRNA, microRNA.

regulation. This effect will result in the programming of a reduced functional capacity of the liver during adult life, affecting its performance and adaptation to metabolic challenges such as HFD or other nutritional insults (14).

Moreover, this "liver metabolic imprinting" during fetal life may further contribute to the pathogenesis of adult complications such as INS resistance and fatty liver $(14,39)$.

On the contrary, the hypothalamic effect of fetal metabolic programming seems to involve a clustered gene network that senses nutrients and modulates neuroendocrine activity, and this phenomenon is more likely an adaptive response. Of course, it is possible to find, however, some common alterations in both situations, and one caveat should be raised; i.e., there might be some bias in these hypothesis-driven research data.

\section{UNEXPLORED AREAS OF RESEARCH: UMBILICAL CORD DNA METHYLATION, CHROMATIN REMODELING, MIRNAs, AND HUMAN FETAL METABOLIC PROGRAMMING}

Exploring changes in the pattern of DNA methylation in the human umbilical cord has a tremendous potential because of the pluripotency of this tissue. Emerging data from human studies shed light on the association between birth weight, epigenetic modifications, and risk of developing MS-related phenotypes in later life. For instance, we explored the status of promoter DNA methylation of three key genes related with MS (PPARGC1A, PPARG, and TFAM) in newborns between both extremes of abnormal fetal growth and their relationship to the mother's prepregnant characteristics (40). Of note, we observed that promoter methylation of PPARGC1A in umbilical cord of newborns was positively correlated with maternal BMI.

A recent study showed that aberrant DNA methylation of INS-like growth factor-2 in umbilical cord blood is associated with overweight or obesity in a multiethnic cohort (41). Another report showed that umbilical cord tissue methylation at particular CpGs of retinoid X receptor- $\alpha$ and endothelial nitric oxide synthase from healthy neonates was associated with childhood fat mass (42).

Altogether, the above-mentioned evidence from human studies might support the role of early-life DNA methylation in the modulation of later development of obesity and related complications. Although these findings do not prove a cause and effect relationship, they provide an initial proof of principle and open new areas of research that will benefit from welldesigned cohort studies.

Epigenetic changes associated with fetal programming are not restricted to methylation of gene promoters. In fact, other modifications play an important role in the modulation of the fetal epigenotype, e.g., methylation of histones and chromatin remodeling, which are considered hallmarks of transcriptional activation. In fact, histone methylation at specific amino acid residues, such as lysine (Lys, $\mathrm{K}$ ), in histone $\mathrm{N}$-terminal tails is an important modification defining chromatin state. Methylations at $\mathrm{H} 3$ lysine $4(\mathrm{H} 3 \mathrm{~K} 4)$ and lysine $36(\mathrm{H} 3 \mathrm{~K} 36)$ appear to be signals of chromatin activation, whereas methylation of $\mathrm{H} 3 \mathrm{~K} 9$ and $\mathrm{H} 3 \mathrm{~K} 27$ seems to be related to chromatin condensation.

Unfortunately, there is scarce information about the role of histone modifications and changes in fetal chromatin structure 


\section{Review | Sookoian etal.}

and human metabolic programming. We have started to explore patterns of histone methylation across the promoter of PPARGC1A (from -320 to $-700 \mathrm{bp}$ from the transcription start site) and TFAM (from -512 to $-930 \mathrm{bp}$ from the transcription start site) in newborns exposed to different prenatal environments. Briefly, we extracted DNA with or without chromatin enrichment in specific histone modifications by chromatin inmmunoprecipitation from the cell nuclei of umbilical cords. In this analysis, we evaluated 50 newborns, including 16 with appropriate weight for their gestational age and 34 representing both the extremes of abnormal fetal growth: SGA $(n=17)$ and LGA $(n=17)$. We used antibodies specific for modified H3, such as H3K4Me3 and H3K9Me3 (Active Motif, Carlsbad, $\mathrm{CA})$. Specific gene abundance in the immunoprecipitated chromatin against the input, nonenriched chromatin was evaluated by real-time quantitative PCR. Of note, we observed that H3K4Me3-related TFAM promoter levels, representing 10\% $(0.10 \pm 0.02)$, are associated with birth weight (Wilks: $0.60, P<$ 0.014 ) independently of mothers' BMI and fetal homocysteine levels despite the fact that H3K4Me3-related TFAM promoter levels correlated with fetal homocysteine levels (Pearson's $R$ : $0.31, P<0.03)$. This finding is remarkable because homocysteine is a modifiable cardiovascular disease risk factor. Of note, plasma homocysteine of the neonate, although positively correlated with mothers' plasma homocysteine (Spearman's $R$ : 0.51, $P<0.001$ ), was higher than in mothers (mothers: 6.18 $\pm 0.32 \mu \mathrm{mol} / \mathrm{l}$ vs. neonates: $9.26 \pm 0.41 \mu \mathrm{mol} / \mathrm{l}, P<0.001$ ) and was inversely correlated with neonate plasma folic acid (Spearman's $R:-0.35, P<0.002$ ), indicating that the methyl group metabolism is central to the fetus and can be regulated by folate supplementation. This phenomenon seems also to affect chromatin around circadian rhythm genes because the H3K9Me3-related CLOCK promoter negatively correlated with newborn folic acid levels (Spearman's $R:-0.35, P<0.037$ ). The process of histone methylation in $\mathrm{K}$ seems to be general because it affects most of the circadian rhythm genes simultaneously. For instance, H3K4Me3 levels around PER1, CLOCK, and BMAL promoters are highly correlated $(R: 0.69, P<$ 0.0000005 and $R: 0.68, P<0.00002$, respectively). Finally, this process seems to be modulated by the homozygosity for the more replicated obesity-associated risk allele of fat mass and obesity associated (FTO), the rs9930506 A, as we had reported for the PPARGC1A (PGC1A) promoter (40).

Finally, unexplored mechanisms of gene expression regulation such as miRNAs, small noncoding RNAs involved in posttranscriptional gene regulation, represent attractive molecular candidates in the modulation of fetal programming. miRNAs exert their biologic functions after binding (in a sequence-specific manner) to the $3^{\prime}$ untranslated region of mRNA targets, facilitating mRNA degradation or translational inhibition. There are isolated but interesting reports about programmed changes in miRNA expression linking early-life nutrition to adult disease. For instance, the article by Ferland-McCollough et al. showed that miRNA-483-3p is upregulated in the adipose tissue in low-birth-weight adult humans and prediabetic adult rats exposed to suboptimal nutrition in early life (43). Another human study showed that the expression of miRNA-16 and miRNA-21 were markedly reduced in SGA infants (44). In addition, an experimental study observed that maternal obesity was associated with fetal muscle miRNA let-7g expression, thereby influencing intramuscular adipogenesis (45).

Finally, we performed a gene network analysis using the web-server application ToppCluster (http://toppcluster.cchmc. org/) for comparative enrichment of the candidate gene list, shown in Supplementary Appendix S1 online, and miRNAs prediction. Of note, among several miRNAs shown in (Figure 6) is the miRNA34a, a novel regulator of $\operatorname{Smad} 4 / \mathrm{TGF} \beta$ signaling (46) associated with the transcriptional regulation of the NAD-dependent deacetylase SIRT1 (47), which regulates stress response pathways and metabolism.

\section{CONCLUSION AND PERSPECTIVES}

In this review, we have used a combination of gene-protein enrichment analysis resources along with functional annotations and protein interaction networks in an integrative approach to better understand the molecular mechanisms underlying fetal metabolic programming and epigenetic modifications.

Although our results a priori assumed published contributions on fetal metabolic programming, it is worth mentioning that text mining has emerged as a powerful instrument to generate new hypotheses (48). Moreover, systems biology approaches similar to the ones used in this article allow scientists to identify hidden connections among known genes or molecular targets and predict novel functional implications among them. Hence, the integration of the current biomedical literature about fetal metabolic programming emerges as an interesting tool never before explored for retrieving biological data that have been reported in any species and any experimental setting and translate them into new questions or new answers of unresolved issues. For instance, new putative explanations emerged about the question of whether in utero overnutrition modulates fetal metabolic programming in the same fashion as that of a maternal environment of undernutrition, suggesting that the mechanisms behind these two fetal nutritional imbalances are different. Moreover, predicted molecular functions suggest that fetal metabolic programming is associated with changes in a complex network of the liver transcriptome that may permanently affect glucose and lipid metabolism. Conversely, early exposure to a nutrition imbalance is associated with an adaptive response of the central neurons of the hypothalamic region that modulate food intake and energy expenditure by changes in hormonal activity.

Furthermore, new knowledge may soon emerge regarding unexplored epigenetic factors such as histone modifications and regulation of gene transcription by miRNAs. Challenging issues for the future are exciting, for instance, the question of whether these findings that emerged from a complex integration of the literature knowledge based primarily on experimental and animal studies can be translated to humans. Previous efforts in biomedical research offer a unique opportunity of translating basic research from the bench to the clinic. 
SUPPLEMENTARY MATERIAL

Supplementary material is linked to the online version of the paper at http://www.nature.com/pr

\section{STATEMENT OF FINANCIAL SUPPORT}

This study was partially supported by grants PICT 2008-1521 and PICT 20100441 (Agencia Nacional de Promoción Científica y Tecnológica) and UBACYT CM04 (Universidad de Buenos Aires). S.S., T.F.G., A.B., and C.J.P. belong to Consejo Nacional de Investigaciones Científicas (CONICET).

Disclosure: The authors declared no conflict of interest.

\section{REFERENCES}

1. Curhan GC, Willett WC, Rimm EB, Spiegelman D, Ascherio AL, Stampfer MJ. Birth weight and adult hypertension, diabetes mellitus, and obesity in US men. Circulation 1996;94:3246-50.

2. Harder T, Rodekamp E, Schellong K, Dudenhausen JW, Plagemann A. Birth weight and subsequent risk of type 2 diabetes: a meta-analysis. Am J Epidemiol 2007;165:849-57.

3. Osmond C, Barker DJ, Winter PD, Fall CH, Simmonds SJ. Early growth and death from cardiovascular disease in women. BMJ 1993;307: 1519-24.

4. Forsén T, Eriksson JG, Tuomilehto J, Osmond C, Barker DJ. Growth in utero and during childhood among women who develop coronary heart disease: longitudinal study. BMJ 1999;319:1403-7.

5. Barker DJ, Bull AR, Osmond C, Simmonds SJ. Fetal and placental size and risk of hypertension in adult life. BMJ 1990;301:259-62.

6. Poston L. Gestational weight gain: influences on the long-term health of the child. Curr Opin Clin Nutr Metab Care 2012;15:252-7.

7. Hales CN, Barker DJ. The thrifty phenotype hypothesis. Br Med Bull 2001;60:5-20.

8. Barker DJ. A new model for the origins of chronic disease. Med Health Care Philos 2001;4:31-5.

9. Barbosa-Silva A, Fontaine JF, Donnard ER, Stussi F, Ortega JM, Andrade-Navarro MA. PESCADOR, a web-based tool to assist text-mining of biointeractions extracted from PubMed queries. BMC Bioinformatics 2011;12:435.

10. You JS, Kelly TK, De Carvalho DD, Taberlay PC, Liang G, Jones PA. OCT4 establishes and maintains nucleosome-depleted regions that provide additional layers of epigenetic regulation of its target genes. Proc Natl Acad Sci USA 2011;108:14497-502.

11. Warde-Farley D, Donaldson SL, Comes O, et al. The GeneMANIA prediction server: biological network integration for gene prioritization and predicting gene function. Nucleic Acids Res 2010;38(Web Server issue):W214-20.

12. Burgueño AL, Gianotti TF, Mansilla NG, Pirola CJ, Sookoian S. Cardiovascular disease is associated with high-fat-diet-induced liver damage and up-regulation of the hepatic expression of hypoxia-inducible factor 1a in a rat model. Clin Sci 2013;124:53-63.

13. Sookoian S, Pirola CJ. Non-alcoholic fatty liver disease is strongly associated with carotid atherosclerosis: a systematic review. J Hepatol 2008; 49:600-7.

14. Burgueño AL, Cabrerizo R, Gonzales Mansilla N, Sookoian S, Pirola CJ. Maternal high-fat intake during pregnancy programs metabolic-syndrome-related phenotypes through liver mitochondrial DNA copy number and transcriptional activity of liver PPARGC1A. J Nutr Biochem 2013;24:6-13.

15. George LA, Zhang L, Tuersunjiang N, et al. Early maternal undernutrition programs increased feed intake, altered glucose metabolism and insulin secretion, and liver function in aged female offspring. Am J Physiol Regul Integr Comp Physiol 2012;302:R795-804.

16. Camm EJ, Martin-Gronert MS, Wright NL, Hansell JA, Ozanne SE, Giussani DA. Prenatal hypoxia independent of undernutrition promotes molecular markers of insulin resistance in adult offspring. FASEB J 2011; 25:420-7.

17. Begum G, Stevens A, Smith EB, et al. Epigenetic changes in fetal hypothalamic energy regulating pathways are associated with maternal undernutrition and twinning. FASEB J 2012;26:1694-703.
18. Chen H, Simar D, Lambert K, Mercier J, Morris MJ. Maternal and postnatal overnutrition differentially impact appetite regulators and fuel metabolism. Endocrinology 2008;149:5348-56.

19. Bouret SG. Leptin, nutrition, and the programming of hypothalamic feeding circuits. Nestle Nutr Workshop Ser Pediatr Program 2010;65:25-35; discussion 35-9.

20. Gemma C, Sookoian S, Alvariñas J, et al. Mitochondrial DNA depletion in small- and large-for-gestational-age newborns. Obesity (Silver Spring) 2006;14:2193-9.

21. Carabelli J, Burgueño AL, Rosselli MS, et al. High fat diet-induced liver steatosis promotes an increase in liver mitochondrial biogenesis in response to hypoxia. J Cell Mol Med 2011;15:1329-38.

22. Sookoian S, Castaño GO, Burgueño AL, Gianotti TF, Rosselli MS, Pirola CJ. The nuclear receptor PXR gene variants are associated with liver injury in nonalcoholic fatty liver disease. Pharmacogenet Genomics 2010;20:1-8.

23. Sookoian S, Castaño G, Gianotti TF, Gemma C, Rosselli MS, Pirola CJ. Genetic variants in STAT3 are associated with nonalcoholic fatty liver disease. Cytokine 2008;44:201-6.

24. Rosselli MS, Burgueño AL, Carabelli J, Schuman M, Pirola CJ, Sookoian S. Losartan reduces liver expression of plasminogen activator inhibitor-1 (PAI-1) in a high fat-induced rat nonalcoholic fatty liver disease model. Atherosclerosis 2009;206:119-26.

25. Sookoian S, Rosselli MS, Gemma C, et al. Epigenetic regulation of insulin resistance in nonalcoholic fatty liver disease: impact of liver methylation of the peroxisome proliferator-activated receptor $\gamma$ coactivator $1 \alpha$ promoter. Hepatology 2010;52:1992-2000.

26. Pirola CJ, Fernández Gianotti T, Burgueño AL, et al. Epigenetic modification of liver mitochondrial DNA is associated with histological severity of nonalcoholic fatty liver disease. Gut 2012; e-pub ahead of print 9 August 2012.

27. Sookoian S, Gemma C, Pirola CJ. Influence of hepatocyte nuclear factor 4alpha (HNF4alpha) gene variants on the risk of type 2 diabetes: a metaanalysis in 49,577 individuals. Mol Genet Metab 2010;99:80-9.

28. Dallmann R, Weaver DR. Altered body mass regulation in male mPeriod mutant mice on high-fat diet. Chronobiol Int 2010;27:1317-28.

29. Sookoian S, Castaño G, Gemma C, Gianotti TF, Pirola CJ. Common genetic variations in CLOCK transcription factor are associated with nonalcoholic fatty liver disease. World J Gastroenterol 2007;13:4242-8.

30. Sookoian S, Gemma C, Gianotti TF, Burgueño A, Castaño G, Pirola CJ. Genetic variants of Clock transcription factor are associated with individual susceptibility to obesity. Am J Clin Nutr 2008;87:1606-15.

31. Turek FW, Joshu C, Kohsaka A, et al. Obesity and metabolic syndrome in circadian Clock mutant mice. Science 2005;308:1043-5.

32. García SI, Pirola CJ. Thyrotropin-releasing hormone in cardiovascular pathophysiology. Regul Pept 2005;128:239-46.

33. Landa MS, García SI, Schuman ML, et al. Knocking down the diencephalic thyrotropin-releasing hormone precursor gene normalizes obesity-induced hypertension in the rat. Am J Physiol Endocrinol Metab 2007;292:E1388-94.

34. García SI, Porto PI, Dieuzeide G, et al. Thyrotropin-releasing hormone receptor (TRHR) gene is associated with essential hypertension. Hypertension 2001;38(3 Pt 2):683-7.

35. Vacher CM, Crépin D, Aubourg A, et al. A putative physiological role of hypothalamic CNTF in the control of energy homeostasis. FEBS Lett 2008;582:3832-8.

36. Liu QS, Wang QJ, Du GH, et al. Recombinant human ciliary neurotrophic factor reduces weight partly by regulating nuclear respiratory factor 1 and mitochondrial transcription factor A. Eur J Pharmacol 2007;563: $77-82$.

37. Gemma C, Sookoian S, Dieuzeide G, et al. Methylation of TFAM gene promoter in peripheral white blood cells is associated with insulin resistance in adolescents. Mol Genet Metab 2010;100:83-7.

38. Gianotti TF, Sookoian S, Dieuzeide G, et al. A decreased mitochondrial DNA content is related to insulin resistance in adolescents. Obesity (Silver Spring) 2008;16:1591-5.

39. Burgueño AL, Carabelli J, Sookoian S, Pirola CJ. The impact of maternal high-fat feeding on liver and abdominal fat accumulation in adult offspring under a long-term high-fat diet. Hepatology 2010;51:2234-5. 
40. Gemma C, Sookoian S, Alvariñas J, et al. Maternal pregestational BMI is associated with methylation of the PPARGC1A promoter in newborns. Obesity (Silver Spring) 2009;17:1032-9.

41. Perkins E, Murphy SK, Murtha AP, et al. Insulin-like growth factor 2/H19 methylation at birth and risk of overweight and obesity in children. J Pediatr 2012;161:31-9.

42. Godfrey KM, Sheppard A, Gluckman PD, et al. Epigenetic gene promoter methylation at birth is associated with child's later adiposity. Diabetes 2011;60:1528-34.

43. Ferland-McCollough D, Fernandez-Twinn DS, Cannell IG, et al. Programming of adipose tissue miR-483-3p and GDF-3 expression by maternal diet in type 2 diabetes. Cell Death Differ 2012;19:1003-12.

44. Maccani MA, Padbury JF, Marsit CJ. miR-16 and miR-21 expression in the placenta is associated with fetal growth. PLoS ONE 2011;6:e21210.
45. Yan X, Huang Y, Zhao JX, et al. Maternal obesity downregulates microRNA let-7g expression, a possible mechanism for enhanced adipogenesis during ovine fetal skeletal muscle development. Int J Obes (Lond) 2012; e-pub ahead of print 22 May 2012.

46. Genovese G, Ergun A, Shukla SA, et al. microRNA regulatory network inference identifies miR-34a as a novel regulator of TGF- $\beta$ signaling in glioblastoma. Cancer Discov 2012;2:736-49.

47. Yamakuchi M. MicroRNA Regulation of SIRT1. Front Physiol 2012; 3:68.

48. Rebholz-Schuhmann D, Oellrich A, Hoehndorf R. Text-mining solutions for biomedical research: enabling integrative biology. Nat Rev Genet 2012;13:829-39.

49. Hooper SD, Bork P. Medusa: a simple tool for interaction graph analysis. Bioinformatics 2005;21:4432-3. 